\title{
Identification of novel plant architecture mutants in barley
}

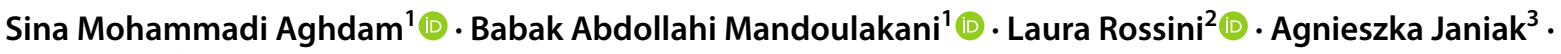 \\ Salar Shaaf ${ }^{2} \mathbb{B}$
}

Received: 15 February 2021 / Accepted: 13 May 2021 / Published online: 27 May 2021

(c) The Author(s) 2021

\begin{abstract}
In grasses, biomass and grain production are affected by plant architecture traits such as tiller number, leaf size and orientation. Thus, knowledge regarding their genetic basis is a prerequisite for developing new improved varieties. Mutant screens represent a powerful approach to identify genetic factors underpinning these traits: the HorTILLUS population, obtained by mutagenesis of spring two-row cultivar Sebastian, is a valuable resource for this purpose in barley. In this study, 20 mutant families from the HorTILLUS population were selected and evaluated for tiller number, leaf angle and a range of other plant architecture and agronomic traits using an unreplicated field design with Sebastian as a check cultivar. Principal Component Analysis revealed strong relationships among number of tillers, upper canopy leaf angle, biomass and yield-related traits. Comparison to the Sebastian background revealed that most mutants significantly differed from the wild-type for multiple traits, including two mutants with more erect leaves and four mutants with increased tiller number in at least one phenological stage. Heatmap clustering identified two main groups: the first containing the two erect mutants and the second containing Sebastian and the high-tillering mutants. Among the high-tillering mutants, two showed significantly higher biomass and grain yield per plant compared to Sebastian. The selected mutants represent promising materials for the identification of genetic factors controlling tillering and leaf angle in barley.
\end{abstract}

Keywords Hordeum vulgare $\cdot$ HorTILLUS · Leaf angle $\cdot$ Tiller number $\cdot$ Biomass

\section{Introduction}

By the year 2030, the global human population will be over 8.3 billion leading to an increase in demands for food and energy (FAO 2017). Development of new crop cultivars with enhanced features is a priority to meet these necessities. Manipulation of plant architecture traits was the most significant result of the Green Revolution, as illustrated by rice and wheat varieties with strong and short stems and higher grain yields (Khush 2001).

Communicated by J. Zimny.

Laura Rossini

laura.rossini@unimi.it

1 Department of Plant Production and Genetics, Faculty of Agriculture and Natural Resources, Urmia University, Urmia, Iran

2 Department of Agricultural and Environmental Sciences (DISAA), University of Milan, Via Celoria 2, Milan, Italy

3 Department of Genetics, University of Silesia in Katowice, 40-032 Katowice, Poland
Barley (Hordeum vulgare ssp. vulgare), an important plant in the history of humanity, has been cultivated for over 10,000 years (Pankin and Von-Korff 2017). Today, barley ranks in fourth place for worldwide cereal production after maize, rice and wheat and third in the European Union (FAOSTAT 2018). Compared to other grasses, barley is highly tolerant to stresses such as drought, cold and salt, which represent key traits for adaptation to climate change (Dawson et al. 2015). Barley grains are mainly used as food, animal feed and malt for the brewing and distilling industries (Munoz-Amatriain et al. 2014). Recently, straw has attracted attention as a source of renewable energy with a view to develop barley as a dual-purpose crop to produce grains and biofuel. Concurrent increase in biomass and grain yield has been proposed as a strategy to meet demands for higher food production (Aisawi et al. 2015). The lack of progress in harvest index increase for the last 40 years makes biomass improvement more important than before (Foulkes et al. 2011).

Among morphological traits contributing to biomass and grain production, leaf angle and tiller number have been 
shown to play an important role and were targeted in several breeding programs (Mock and Pearce 1975; Shearman et al. 2005; Peng et al. 2008).

Leaf angle-i.e. the inclination between leaf blade midrib and stem-is a major trait influencing light interception, photosynthetic efficiency throughout the canopy and productivity in relation to planting density (Mantilla-Perez and Salas Fernandez 2017). Optimal plant architecture would require erect leaves on the upper part and horizontally arranged ones in the lower part of the canopy, as suggested in the smart canopy concept for crop biomass and yield (Ort et al. 2015). For example, in winter and spring wheat leaf angle was shown to affect photosynthetic efficiency and net carbon accumulation and a higher rate of dry matter production has been reported for spring wheat genotypes with erect leaves (Choudhury 2000; Parry et al. 2011). In rice, smaller leaf angle was also reported as a substantial morphological factor contributing to higher biomass production ( $\mathrm{Li}$ et al. 2009; Sakamoto et al. 2006; Kumagai et al. 2014).

Another important character influencing yield and yield components in wheat, rice and barley is tillering (Sakamoto and Matsuoka 2004; Sreenivasulu and Schnurbusch 2012; Shaaf et al. 2019). Tillers are branches arising from basal nodes of the culm, affecting shoot architecture and consequently crop density and biomass (Donald 1968; Seavers and Wright 1999). In rice and other grasses, a positive correlation between tiller number and biomass has been demonstrated (Barnaby et al. 2019), however excessive tillering can negatively affect grain filling. Hence, a moderate increase in tiller number is preferable to improve biomass and yield (Boe 2007; Das et al. 2004).

As supported by these examples, knowledge regarding the genetic basis of plant architecture traits and their relationships with biomass and yield is necessary for crop improvement. However, compared to rice, relatively few genes controlling leaf angle and tillering have been identified in barley (reviewed in Shaaf et al. 2019) and their relationships with grain and biomass production is not well studied. Mutagenized populations are powerful tools to dissect the genetic bases of traits and identify the underlying genes (Ahloowalia et al. 2004; Nachimuthu et al. 2015; Riaz and Gul 2015). Barley benefits from large mutant collections that were successfully used in previous studies to discover genes involved in tillering and leaf angle (Druka et al. 2011; Tavakol et al. 2015; Liller et al. 2015; Mascher et al. 2014; Dockter et al. 2014). Several mutants with erect leaf angle have been reported such as uzul, ligl, ari-e, ari-o, ari-u.245, brdl, brh1, brh13.p and, brd1-3, brh2 and ari-l (reviewed in Shaaf et al. 2019). Among these, the $u z u l$ mutant was widely used to develop cultivated barley varieties with erect leaves, semidwarf stature, increased lodging resistance and yield (Chono et al. 2003). On the other hand, Braumann et al. $(2017 ; 2018)$ showed reductions in grain yield and thousand-grain-weight for lines carrying mutations in Brhl or Brh2, respectively, indicating that erect leaf mutants vary in terms on agronomic performance, also depending on genetic background.

Previously described barley high-tillering mutants include granum-a (gra-a), many noded dwarfl (mndl), mnd3, mnd6 and grassy tillers, hvd14, intermedium spike-c (int-c), int-m, semidwarfl ( $s d w 1)$ (reviewed in Shaaf et al. 2019). Jia et al. (2011) reported significant increase of tiller number and yield in the $s d w 1$ mutant, however other reports suggest that this effect may depend on genetic background and/or environmental conditions (reviewed in Kuczynska et al. 2013). Another high tillering mutant int- $m$ exhibited reduced number of seeds compared to wild type (Liller et al. 2015). Chemical mutagenesis is a powerful approach for the development of mutagenized populations that can be used in forward and reverse genetics approaches to screen for mutants with desired phenotypes. In the last two decades several Targeting Induced Local Lesions in Genomes (TILLING) populations have been produced for barley (Caldwell et al. 2004; Talamè et al. 2008; Gottwald et al. 2009; Lababidi et al. 2009; Lundqvist 2014; Schreiber et al. 2019). Among them, an interesting resource is HorTILLUS (Hordeum vulgare-TILLING-University of Silesia), developed from double treatment of spring barley variety "Sebastian" with sodium azide (NaN3) and N-Methyl-Nnitrosourea (MNU) (Szurman-Zubrzycka et al. 2018). Its high average mutation density $(1 / 477 \mathrm{~kb}$, calculated on the basis of 32 genes) makes HorTILLUS an ideal tool for both functional genomic studies and forward selection of barley mutants with required phenotypic changes. In the current investigation, new mutants altered in leaf angle and tiller number were identified from the HorTILLUS population and characterized. Relationships with yield, yield components and biomass along with other traits were also studied.

\section{Materials and methods}

\section{Plant materials and initial screening}

The genetic materials used for the present work were selected from the HorTILLUS population derived from sodium azide $\left(\mathrm{NaN}_{3}\right)$ and $N$-Methyl- $N$-nitrosourea (MNU) mutagenesis of the barley spring two-row cultivar "Sebastian" (Szarejko and Maluszynski 1999; Szarejko et al. 2017; Szurman-Zubrzycka et al. 2018). This cultivar was selected due to its high yield potential, malting quality and resistance to several important diseases. $\mathrm{M}_{3}$ seeds from a subset of 3481 individual $\mathrm{M}_{2}$ plants with the highest number of seeds were planted in the field and phenotypically evaluated (Szurman-Zubrzycka et al. 2018). Progenies of these plants were used in the present study to select mutants with increased tiller number (TN) and erect leaf angle (LA). The 
screening was performed in two batches in 2015 and 2016 at the experimental field of University of Silesia in Katowice, Poland, located in Bochuchwalowice village (50 26 47.6" $\mathrm{N} 19^{\circ} 11^{\prime} 05.1^{\prime \prime} \mathrm{E}$ ). $\mathrm{M}_{4}$ seeds from $854 \mathrm{M}_{3}$ families (375 families in 2015 and 479 families in 2016) were used for the screening. Seeds of each family were evenly sown in two rows of $120 \mathrm{~cm}$ length and $15 \mathrm{~cm}$ spacing, 10 seeds per row (altogether 20 seeds per $\mathbf{M}_{3}$ family). Seeds of Sebastian cultivar were sown in 2-row replicates every 30 rows between the mutagenized lines. The phenotypic evaluation was performed at the heading stage. In 2015, the leaf angle was evaluated by visual comparison of Sebastian and mutant lines. Additionally, the angle between external leaves was determined: this angle for Sebastian cultivar was on average $90^{\circ}$, thus all mutant plants with narrower angle were considered as more erect and selected for further analysis. In 2016 all candidate lines selected the previous year together with 479 newly sown families were examined for insertion angle of penultimate leaf (leaf below the flag leaf) from three tillers using the PocketPlant3D smartphone application (Confalonieri et al. 2017). Tiller number was counted manually in both years of screening, at heading stage. Altogether around 14,400 individual $M_{4}$ plants were subjected to phenotyping. A total of 75 candidate mutant plants from $24 \mathrm{M}_{4}$ families were selected for further analysis as potentially interesting for LA and TN.

\section{Field evaluation and phenotyping}

Twenty $\mathrm{M}_{5}$ or $\mathrm{M}_{6}$ seeds from selected individual plants of each mutant family were sown at the end of February 2017 at "F. Dotti" experimental farm from Università degli Studi di Milano, Lodi, Italy ( $45^{\circ} 20^{\prime} 18.7^{\prime \prime} \mathrm{N} 9^{\circ} 27^{\prime} 03.6^{\prime \prime} \mathrm{E}$ ) using an unreplicated field design in three blocks containing the wild-type Sebastian as check cultivar. Finally, a total of 20 mutant families were selected for further screening and evaluation of phenotypic stability and performance, after excluding 4 families due to germination problems (Table 1). The field was plowed at $20 \mathrm{~cm}$ depth and before sowing the seedbed was prepared for better germination. Seeds from 2015- and 2016-selected plants of each mutant were sown in each plot with two rows containing ten seeds within each row. The distance between plants within rows was $14 \mathrm{~cm}$ and $30 \mathrm{~cm}$ was the spacing between rows. The winter bread wheat cultivar Bologna was grown between barley plots (equal distances of $30 \mathrm{~cm}$ from barley rows). Sebastian was replicated also in 28 plots distributed across the field experiment. Weeds were controlled by hand at tillering and stem elongation stages. Data were collected from at least 10 plants per family for 26 phenological and agronomical characters at different development stages as follows (See supplementary Table S1 for additional information).
At the tillering stage, we measured tiller number (TNT), 50 days after sowing when plants were still prostrate. At heading stage, we measured days to heading (DH), number of tillers at heading (TNH), and the leaf insertion angle (PAngle) using smartphone application PocketPlant3D (Confalonieri et al. 2017). At ripening stage, we measured days to ripening (DR), plant height $(\mathrm{PH})$, total number of tillers at ripening (TNR), fertile tiller number (FTNR), peduncle extrusion (PedE), flag leaf length (FLL), spike length $(\mathrm{SpL})$, awn length (AwnL), and spike width $(\mathrm{SpW})$. Each plant was finally harvested and the following traits were measured at the post-harvest stage: peduncle length (PedL), length of first uppermost internode below the peduncle (Int 1L), length of the second internode below the first internode (Int2L), third internode length (Int3L), fourth internode length (Int4L), fifth internode length (Int5L), sixth internode length (Int6L), spike weight (SpWt), seed yield per plant (SeedYPl), harvest index (HI), number of seeds per spike (SeedSp), number of seeds per plant (SeedPl), and aboveground biomass per plant.

\section{Statistical analyses}

All statistical analyses were performed in Rstudio (RStudio Team 2020). Analysis of variance was conducted using a linear mixed model implemented in the R package lme4, with mutants as fixed and blocks and lines within mutants as random effects (Bates et al. 2015). The adjusted means of the mutants and standard errors were then calculated using R package "emmeans". Means of each mutant were then compared to control cultivar Sebastian for each trait and their difference was tested using the "multcomp" $\mathrm{R}$ package and raw p-values were adjusted using the false discovery rate (FDR) method. For subsequent analysis the adjusted means of mutants and Sebastian for each trait were used. A hierarchical clustering heatmap of mutants by traits was drawn using R package "heatmaply" after linear normalization of the phenotypic data $(x=y$-min/ max-min) in which $x$ values were encoded as a grid of colored cells. The rows and columns of the matrix were ordered to highlight patterns and accompanied by dendrograms. Pairwise Spearman rank correlation coefficients of variables were calculated and the resulting matrix of coefficients was visualized as a heatmap highlighting clusters of intercorrelated traits. To reduce the complexity of relationships, a data reduction technique was performed using principal components analysis (PCA) implemented in the $\mathrm{R}$ package "factoextra" and the resulting PC scores were plotted. To know the relative importance of each variable on each PC score a loading plot of PCs was visualized using the R package "qgraph". 
Table 1 Mean values for evaluated mutants measured for 26 traits

\begin{tabular}{|c|c|c|c|c|c|c|c|c|c|c|c|c|c|c|}
\hline Group & Genotype & PAngle & TNT & $\mathrm{TNH}$ & TNR & FTNR & DH & DR & PedE & FLL & $\mathrm{PH}$ & $\mathrm{SpL}$ & AwnL & SpW \\
\hline Sebastian & Sebastian & 16.22 & 7.95 & 14.26 & 17.08 & 10.34 & 150 & 179 & -8.88 & 7.73 & 41 & 9.66 & 12.76 & 0.924 \\
\hline Other & M01.0787 & 12.4 & 4.64 & $\underline{8.57}$ & 9.97 & $\underline{5.3}$ & $\underline{154}$ & 179 & -8.95 & $\underline{5.19}$ & $\underline{32.3}$ & $\underline{7.53}$ & 11.35 & $\underline{0.841}$ \\
\hline Other & M02.0509 & 12.21 & 7.61 & 9.6 & 11 & 7.99 & 147 & 175 & $=6.76$ & 5.96 & $\underline{47.7}$ & 8.06 & 9.39 & 0.92 \\
\hline Other & M03.1687 & 10.29 & 7.61 & 11.98 & 12.98 & 9.26 & 154 & 179 & -9.57 & 5.77 & 34 & 8.12 & 11.93 & 0.974 \\
\hline Other & M04.2143 & 10.5 & 8.8 & 14.94 & 22.96 & 9.91 & $\underline{154}$ & 184 & -8.28 & 7.23 & $\underline{26.1}$ & $\underline{6.25}$ & $\underline{10.34}$ & 0.987 \\
\hline LA & M05.5061 & 9.76 & 6.44 & $\underline{10.73}$ & 14.28 & 9.19 & $\underline{136}$ & $\underline{164}$ & -6.24 & $\underline{5.83}$ & 39 & 8.46 & 12.75 & $\underline{0.856}$ \\
\hline LA & M06.7165 & $\underline{5.44}$ & 6.36 & 15.06 & 19.57 & 12.26 & 149 & 180 & -6.34 & $\underline{4.76}$ & $\underline{31.7}$ & 8.54 & $\underline{6.8}$ & $\underline{0.983}$ \\
\hline $\mathrm{TN}$ & M07.5478 & $\underline{24.38}$ & $\underline{13}$ & $\underline{26.76}$ & $\underline{30.05}$ & $\underline{18.13}$ & 148 & 178 & -6.84 & 8.53 & $\underline{47.8}$ & 9.51 & $\underline{10.86}$ & 0.958 \\
\hline $\mathrm{TN}$ & M08.0589 & $\underline{18.78}$ & 11.4 & $\underline{17.95}$ & $\underline{22.11}$ & $\underline{13.94}$ & $\underline{147}$ & $\underline{175}$ & -8.3 & 8.26 & $\underline{46.1}$ & $\underline{10.79}$ & 12.8 & 0.94 \\
\hline $\mathrm{TN}$ & M09.7479 & 18.71 & 7.68 & $\underline{17.34}$ & $\underline{21.15}$ & 12.34 & $\underline{153}$ & 179 & -10.85 & 8.11 & 38.1 & 10.16 & 12.21 & $\underline{0.868}$ \\
\hline Other & M10.0908 & 18.26 & 8.04 & 13.9 & 17.97 & 10.26 & 151 & 179 & -10.2 & 8.34 & 37.5 & 9.81 & 12.94 & 0.909 \\
\hline Other & M11.2167 & 15.04 & 7.55 & 13.3 & 16.1 & 9 & $\underline{154}$ & 182 & -10.04 & 6.99 & $\underline{34.7}$ & 9.45 & 12.91 & 0.919 \\
\hline Other & M12.2610 & 12.63 & 7.88 & 14.14 & 17.35 & 8.51 & $\underline{154}$ & 182 & -8.83 & 6.8 & 35.9 & 9.19 & 11.47 & 0.879 \\
\hline Other & M13.2616 & 12.29 & 6.44 & 11.75 & 13 & 7.01 & 150 & 177 & -8.29 & 7.47 & 42.2 & 9.11 & 12.01 & 0.813 \\
\hline Other & M14.2753 & 14.73 & 6.68 & 11.29 & 13.08 & 6.82 & $\underline{153}$ & 180 & -10.56 & 6.49 & 38.5 & 8.78 & 12.23 & $\underline{0.817}$ \\
\hline Other & M15.2848 & 14.85 & 7.07 & 15.04 & 19.01 & 8.53 & $\underline{153}$ & $\underline{185}$ & -6.28 & $\underline{5.52}$ & $\underline{28.1}$ & $\underline{7.9}$ & $\underline{11.03}$ & $\underline{0.778}$ \\
\hline $\mathrm{TN}$ & M16.3026 & 15.91 & 8.73 & $\underline{17.71}$ & 19.7 & 11.7 & 150 & 179 & -8.48 & 8.38 & 39.8 & 9.66 & 12.84 & 0.924 \\
\hline Other & M17.5022 & 14.37 & 8.08 & 16.04 & 19.51 & 12.5 & 148 & 180 & -8.64 & 7.77 & 42.1 & 10.58 & 12.58 & 0.946 \\
\hline Other & M18.5267 & 17.65 & 7.91 & 17.84 & 21.79 & 12.34 & 148 & 176 & -7.44 & 9.31 & 45.7 & 10.1 & 11.73 & 0.917 \\
\hline Other & M19.5306 & $\underline{19.77}$ & 7.86 & 14.49 & 17.79 & 10.22 & 150 & 178 & $=10.36$ & 8.06 & 37.1 & 10.04 & $\underline{11.99}$ & $\underline{0.975}$ \\
\hline \multirow[t]{2}{*}{ Other } & M20.0161 & 16.51 & 6.59 & 11.36 & 12.93 & 7.02 & $\underline{154}$ & 172 & -9.79 & 9.85 & $\underline{32.5}$ & 8.74 & $\underline{13.49}$ & 0.923 \\
\hline & & Int6L & Int5L & Int $4 \mathrm{~L}$ & Int3L & Int2L & Int1L & PedL & SeedSp & SeedPl & SpWt & SeedYPl & Biomass & $\mathrm{HI}$ \\
\hline Sebastian & Sebastian & 0.869 & 1.97 & 3.74 & 5.4 & 6.94 & 10.78 & 11.27 & 26.3 & 210.3 & 1.641 & 11.7 & 22.1 & 50.8 \\
\hline Other & M01.0787 & 0.879 & 1.83 & 3.14 & $\underline{4.23}$ & $\underline{5.48}$ & $\underline{8.58}$ & $\underline{7.9}$ & 20.1 & $\underline{86.1}$ & $\underline{1.043}$ & $\underline{4.02}$ & $\underline{8.9}$ & 44.4 \\
\hline Other & M02.0509 & $\underline{1.724}$ & $\underline{3.28}$ & $\underline{\mathbf{5 . 3 3}}$ & $\underline{6.51}$ & $\underline{7.89}$ & 11.16 & 11.24 & 21.7 & 146.8 & 1.051 & 6.55 & 13.5 & 47.9 \\
\hline Other & M03.1687 & 0.777 & 1.87 & 3.39 & 4.73 & 5.8 & 9.03 & 8.56 & 24.8 & 189.4 & 1.38 & 9.96 & 19.2 & 51 \\
\hline Other & M04.2143 & $\underline{0.425}$ & 1 & $\underline{2.04}$ & $\underline{2.73}$ & $\underline{3.37}$ & $\underline{6.99}$ & 9.37 & $\underline{14.1}$ & 96.3 & $\underline{0.831}$ & 5.64 & 16.7 & $\underline{32.7}$ \\
\hline LA & M05.5061 & $\underline{0.414}$ & $\underline{1.43}$ & 3.19 & 4.86 & 6.41 & 10.1 & $\underline{13.16}$ & 22.9 & 153.3 & 1.287 & 7.36 & 13.7 & $\underline{54.1}$ \\
\hline LA & M06.7165 & 0.971 & 2 & 3.2 & $\underline{4.4}$ & $\underline{5.69}$ & $\underline{7.85}$ & $\underline{7.49}$ & 24 & 207.4 & $\underline{1.06}$ & 8.24 & 17.3 & 48 \\
\hline $\mathrm{TN}$ & M07.5478 & 1.167 & 2.42 & 4.14 & 5.68 & $\underline{7.78}$ & $\underline{12.71}$ & $\underline{13.18}$ & $\underline{28.6}$ & $\underline{360.5}$ & 1.673 & $\underline{17.89}$ & 35.3 & 52.8 \\
\hline $\mathrm{TN}$ & M08.0589 & 0.964 & 2.1 & $\underline{4.23}$ & $\underline{6.05}$ & $\underline{7.78}$ & $\underline{12.11}$ & $\underline{12.84}$ & $\underline{28.9}$ & $\underline{295.9}$ & $\underline{1.853}$ & $\underline{16.67}$ & $\underline{31}$ & 51.7 \\
\hline $\mathrm{TN}$ & M09.7479 & 0.729 & $\underline{1.68}$ & 3.36 & 5.45 & 7 & 9.9 & 9.9 & 25.5 & $\underline{235.9}$ & 1.423 & 11.33 & 23.4 & 48.3 \\
\hline Other & M10.0908 & 0.844 & 1.86 & 3.4 & 4.91 & 6.49 & 10 & 10.23 & 21 & 158.8 & 1.416 & 9.43 & 21.3 & 43.9 \\
\hline Other & M11.2167 & 0.86 & 1.71 & 2.99 & 4.43 & 5.82 & 9.02 & 9.69 & 22.1 & 155.6 & 1.346 & 8.06 & 17.1 & 45.9 \\
\hline Other & M12.2610 & 0.836 & 1.73 & 3.31 & 4.78 & 6.1 & 9.35 & 9.46 & 21.4 & 151.5 & 1.253 & 8.39 & 19.2 & 41.6 \\
\hline Other & M13.2616 & 0.77 & 2.04 & 3.77 & 5.67 & 7.26 & 11.02 & 11.77 & 23.9 & 126.9 & 1.33 & 5.9 & 12.2 & 46.2 \\
\hline Other & M14.2753 & $\underline{1.234}$ & 2.3 & 3.71 & 5.14 & 6.18 & 9.29 & 10.25 & $\underline{19.3}$ & 96.8 & $\underline{1.044}$ & $\underline{4.53}$ & 12.3 & $\underline{37.7}$ \\
\hline Other & M15.2848 & $\underline{0.46}$ & $\underline{1.08}$ & $\underline{1.72}$ & $\underline{2.85}$ & $\underline{4.43}$ & $\underline{7.94}$ & $\underline{9.55}$ & $\underline{15.8}$ & $\underline{95.4}$ & $\underline{0.852}$ & $\underline{4.65}$ & $\underline{12.2}$ & $\underline{37.6}$ \\
\hline $\mathrm{TN}$ & M16.3026 & 0.761 & 1.75 & 3.44 & 5.1 & 6.66 & 10.74 & 11.42 & 25.5 & 222.3 & 1.636 & 12.01 & 24 & 48 \\
\hline Other & M17.5022 & 0.852 & 1.95 & 3.66 & 5.37 & 7 & 11.34 & 11.74 & 20.7 & 218.8 & 1.395 & 12.73 & 26.9 & 44 \\
\hline Other & M18.5267 & 1.054 & $\underline{2.59}$ & $\underline{4.53}$ & $\underline{6.08}$ & 7.47 & 11.63 & $\underline{12.74}$ & 27.2 & 204.5 & 1.679 & 11.1 & 25.8 & 42.6 \\
\hline Other & M19.5306 & 0.823 & 1.84 & 3.73 & 5.46 & 6.82 & 9.16 & 9.31 & 22.6 & 153.6 & 1.549 & 9.82 & 20.8 & 46.8 \\
\hline Other & M20.0161 & 0.655 & 1.48 & $\underline{2.69}$ & $\underline{4.12}$ & $\underline{5.45}$ & 8.69 & 9.5 & 22.8 & 120.5 & 1.113 & 5.82 & 12.6 & 47.4 \\
\hline
\end{tabular}

Values with significant higher and lower values compared to Sebastian are underlined with bolded and italic text, respectively (see Supplemental Fig. S1) 


\section{Results}

\section{Screening of the HorTILLUS population for leaf angle and tillering mutants}

Comparison to the Sebastian background revealed that most mutants significantly differed from the wild-type for multiple traits (Table 1, Figure S1). Considering the intended purpose of identifying mutants with erect leaves and increased tillering, six mutants emerged as potentially interesting. Two mutants exhibited more erect leaves compared to Sebastian (LA mutants), although they differed with respect to other traits. Mutant M05.5061 displayed lower values for PAngle, TNH, DH, DR, FLL, SpW, and higher HI, PedE, PedL. Mutant M06.7165 had lower PAngle, FLL, AwnL and SpWt, higher SpW; PH was also reduced in this mutant as a result of decreased Int $1 \mathrm{~L}$, Int2L, Int3L, PedL.

Four mutants produced more tillers than Sebastian in at least one of the growth stages (TN mutants). Mutants M07.5478 and M08.0589 had increased tillers for TNT, TNH, TNR, and FTNR. Mutant M09.7479 had increased tillers at heading and ripening, but not at tillering stage. One mutant, M16.3026, showed increased tiller number only at heading stage (TNH). M07.5478 had increased PAngle, PH, PedE, SeedSp, SeedPl, SeedYPl and Biomass, while showing a lower value for AwnL. M08.0589 showed higher values for PAngle, PedL, PH, SpWt, SpL, SeedSp, SeedPl, SeedYPl, Biomass, and lower values for DH and DR compared to Sebastian. Mutant M09.7479 from this group had higher values for DH and SeedPl, and lower values for $\mathrm{SpW}$, PedE.

Of the 14 remaining lines, four (M03.1687, M10.0908, M17.5022 and M13.2616) exhibited no statistically significant differences compared to the wild-type (partly due to variation within families), while 10 differed from Sebastian for various traits. Mutant M01.0787 showed significantly lower values for traits TNT, TNH, TNR, FTNR, FLL, PH, SpL, SeedPl, SpWt, SeedYPl and Biomass, while DH was higher compared to Sebastian. Mutant M02.0509 had higher $\mathrm{PH}$ and lower AwnL compared to Sebastian variety. Traits PH, SpL, AwnL, SeedSp, SpWt and HI had significantly lower values for mutant M04.2143 compared to Sebastian, while DH value was higher. Mutant M11.2167 showed a significantly higher value for DH but lower values for PH. Trait DH was the only significant difference for mutant M12.2610 compared to wild-type. Mutant M14.2753 showed higher values for DH, and lower values for traits PedE, SeedSp, SpW, SpWt, SeedYPl and HI. For mutant M15.2848 higher values for DH, DR, PedE and lower values for FLL, PH, PedL, SpL, AwnL, SeedSp, SeedPl, SpW, SpWt, SeedYPl, biomass and HI were observed. Higher values of PedL were observed for mutant M18.5267 compared to Sebastian

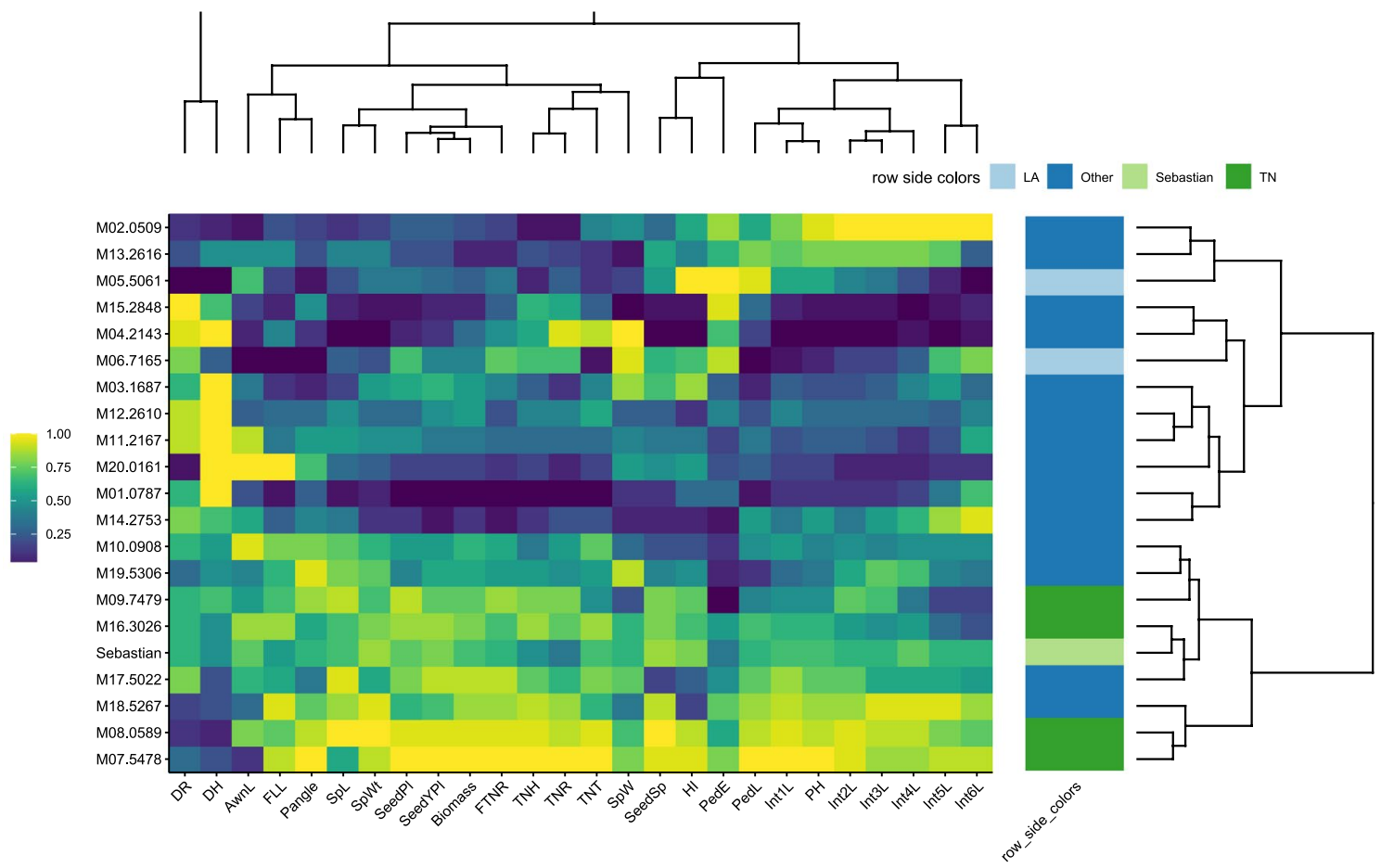

Fig. 1 Heatmap of two-dimensional visualization of genotypes-by-trait table based on normalized values encoded as a grid of colored cells. The rows and columns of the matrix are ordered to highlight patterns accompanied by dendrograms 
variety. For traits PAngle, SpW higher values were observed for mutant M19.5306, while AwnL, PedE, PedL had lower values. Mutant M20.0161 showed significant increases for DH, FLL and AwnL and a lower value for PH compared to Sebastian variety.

Based on heatmap clustering, the 21 genotypes were split into two major groups (Fig. 1). The first cluster includes 12 mutants, two LA and 10 other mutants. The group is characterized by lower values for most traits except for high values of internode length, DH, DR, SpW and HI. Mutant M05.5061 from LA group showed the highest value for $\mathrm{HI}$ among all mutants. The second cluster is a smaller group consisting of all TN mutants, four other mutants and Sebastian suggesting a closer phenotypic similarity to the background compared to the first group. This group is characterized by higher values for most yield and biomass traits especially for tiller number at different stages. For example, mutant M07.5478 had the highest values for tiller number in different stages, PAngle, $\mathrm{PH}$, SeedPl, SeedYPl and Biomass, while M08.0589 exhibited the highest values of SpL, SpWt, and SeedSp.
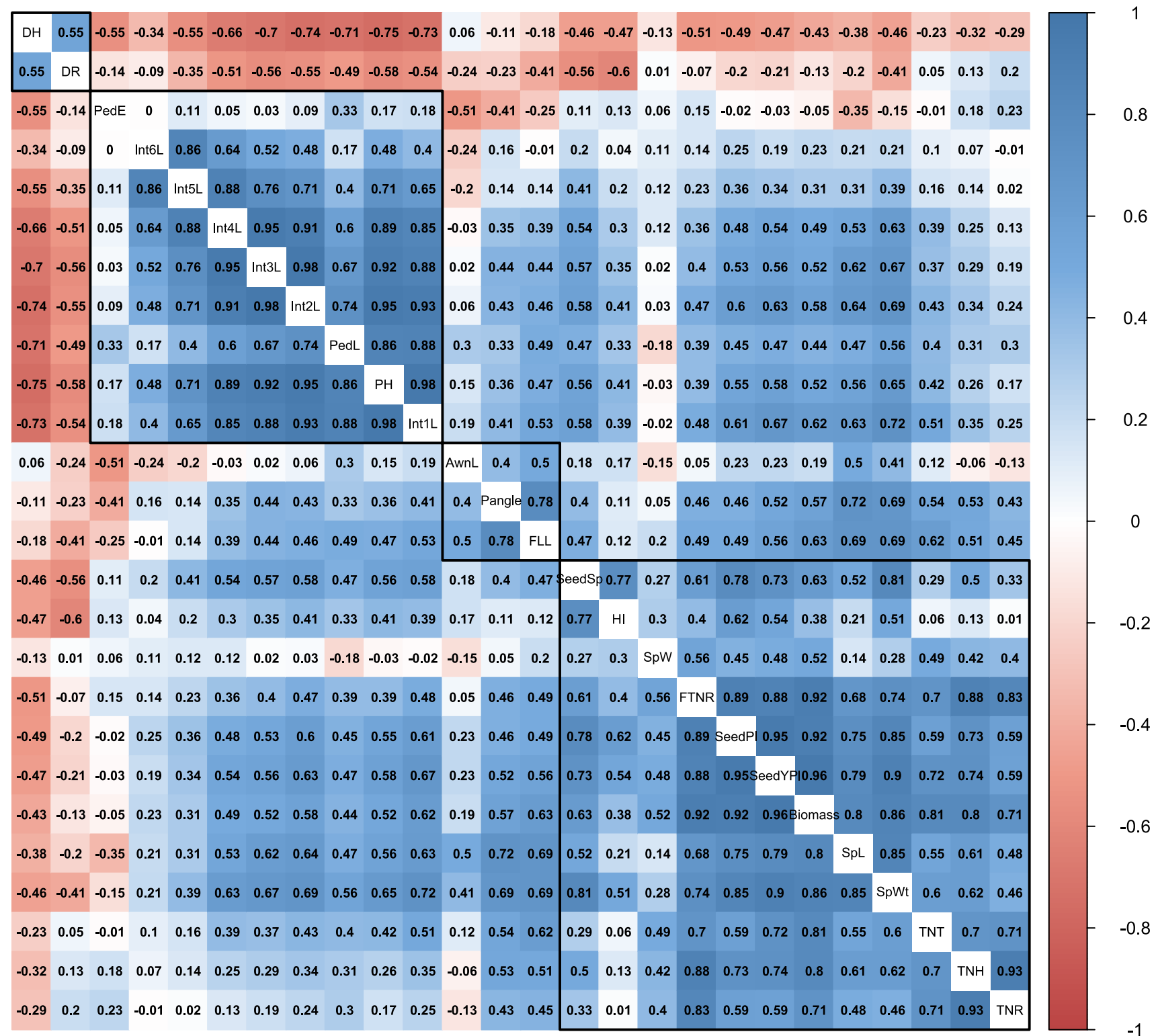

Fig. 2 Plot of correlogram showing pairwise Spearman correlations between 26 traits evaluated on 20 mutants from HorTILLUS population and background cultivar Sebastian. The frames indicate traits with stronger intracorrelations and the colors show negative or positive values based on the scale bar 


\section{Trait correlations}

The correlation coefficients between each pair of traits are presented in Fig. 2. The correlogram revealed four clusters of traits with positively stronger correlations within-cluster. For example, DH and DR are strongly and positively
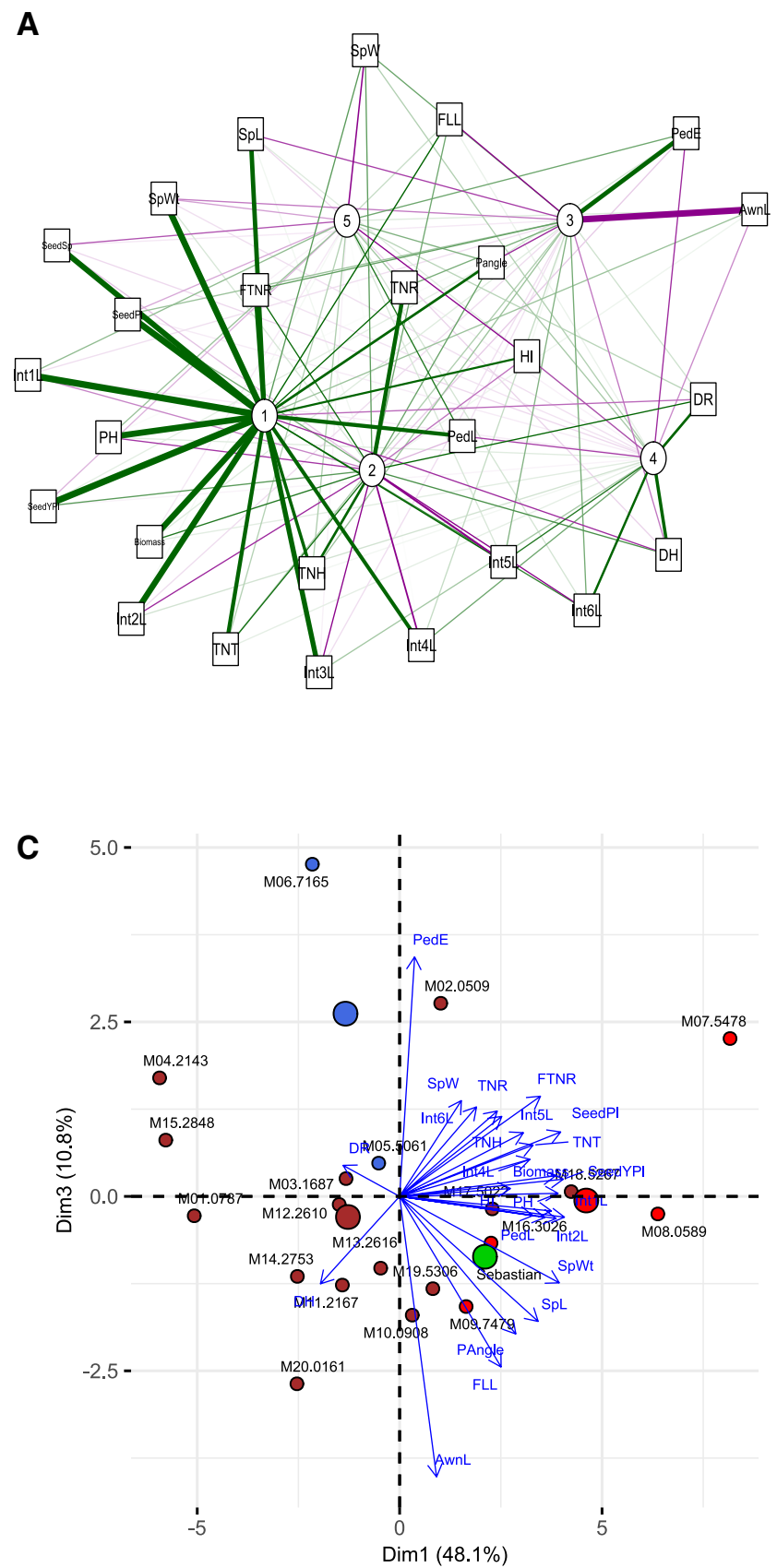

Fig. 3 Visualization Principal Component Analysis (PCA) for 26 traits evaluated on 20 mutants with background cultivar Sebastian. a Loading plot of traits on first five PC scores, green and red edges indicate positively and negatively correlated traits on the corresponding PC scores, respectively. The width of edges indicates the strength of relationship; Biplot of PC1 versus PC2 (b), PC1 versus PC3 (c), correlated, but show predominantly negative correlations with traits from other clusters. PAngle had strong positive correlations with AwnL and FLL. In the larger intra-correlation cluster, all biomass- and yield-related traits were present with stronger positive correlations with tiller number traits. Also, PAngle showed a moderate correlation with biomass
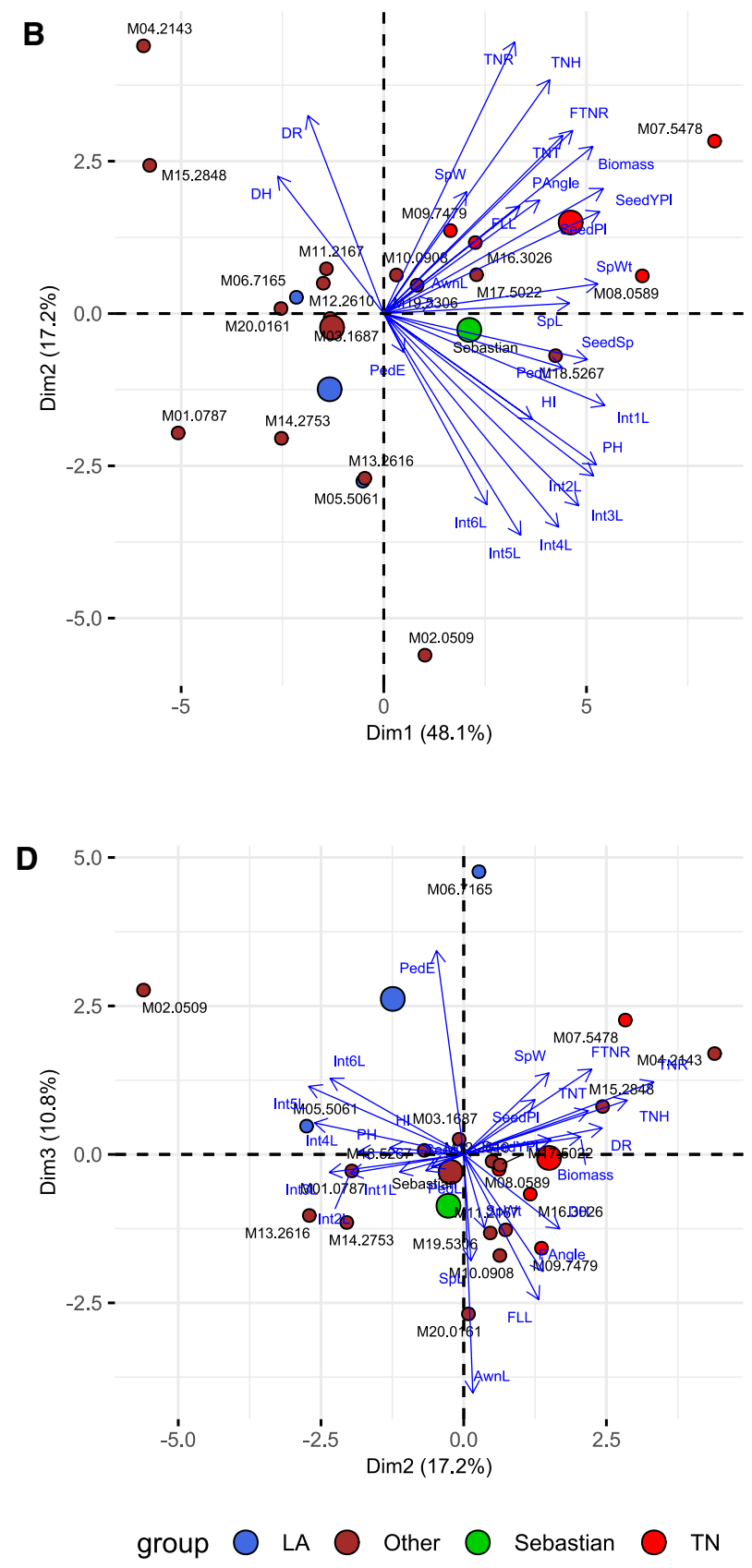

and PC2 versus PC3 (d), with vectors pinned at the origin of PCs. Their projected values on each PC show how much weight they have on that PC. Major groups are color coded. The large circles represent the group summaries (the median PC projection for each of the groups) 
and SeedYPl. These results indicate a positive relationship of tiller number in different stages and penultimate leaf angle with biomass.

\section{Principal components analysis}

The loading plot of traits on the first five PCs is illustrated in Fig. 3a. In the plot, green and purple edges show positive and negative correlations, respectively, between traits (boxes) and PCs (circles). The wider the edge, the stronger the correlation and vice versa. PCA analysis revealed that the main traits contributing to $\mathrm{PC} 1$ are biomass, tiller number, plant height and internode length. The main traits contributing to PC2 are TNR and TNH. PedE and AwnL respectively showed positive and negative contributions to PC3. Days to heading and ripening were the major loadings on PC4.

PCA analysis indicated that the first four PCs with eigenvalues higher than one were the most important PCs explaining, respectively, $48.1 \%, 17.2 \%, 10.8 \%$, and $8.7 \%$ of total variance, and with a cumulative variance of $84.7 \%$ (Fig. 3). In the biplot of PC1 vs. PC2 (Fig. 3b), the first principal component separated LA mutants from $\mathrm{TN}$ and Sebastian, while the second principal component could distinguish both LA and TN groups from Sebastian: mutants M07.5478 and M08.0589 belonging to the TN group, possessed greater values of biomass- and yield-related traits. In the second biplot of PC1 versus PC3 (Fig. 3c), M06.7165 could be distinguished from other mutants mainly because of reduced AwnL and increased PedE. The biplot of PC1 vs. PC4 (Fig. 3d) allowed separation of LA mutant M05.5061 from others due to its early heading and ripening. Compared to other mutants, M09.7479 and M16.3026 were phenotypically closer to Sebastian.

\section{Discussion}

Abundant mutant collections are available for barley and have been exploited to identify a plethora of morphological mutants. However, most previous mutant screenings have only considered few traits, lacking information about other agronomic traits. In the current study, 20 barley families from the HorTILLUS population were evaluated under field conditions for a total of 26 plant architecture, biomass and yield-component traits taking a multivariate analysis approach in order to comprehensively profile each mutant and explore relationships among different traits.

Among them, two mutants exhibited significantly more erect PAngle, and four mutants had higher tiller numbers. The latter in general demonstrated higher biomass and yield related characteristics.

LA mutant M06.7165 exhibited erect leaves, reduced plant height, awn length, and spike weight, while biomass and seed yield per plant were comparable to Sebastian. This mutant is phenotypically similar to the ari-e mutant, caused by a defective allele of the $H v D E P 1$ gene encoding a AAG3-type gamma subunit of heterotrimeric $\mathrm{G}$ protein (Wendt et al., 2016). The ari-e mutation was uncovered in the Scottish malting barley cultivar Golden Promise and shown to result in erect leaf angle, reduced plant height and awn length: agronomic data collected from multiple trials showed that this allele can produce either positive or negative effects on grain yield depending on the environment and genetic background (Wendt et al. 2016). It would be interesting to test whether such factors also affect the performance of M06.7165.

LA mutant M05.5061 was characterized by the highest harvest index, early heading and ripening. In contrast to the reduced plant height of many erect mutants (Dockter et al. 2014), the overall plant height of M05.5061 is comparable to Sebastian. Interestingly, basal internodes were shorter in the mutant, but peduncle length was increased, as was peduncle extrusion. Taken together these features distinguish this mutant from other erect leaf mutants, making it an interesting target for further genetic analysis.

Considering the high plasticity of tillering throughout the plant life cycle, we decided to record data for tiller numbers at different phenological stages (tillering, heading and ripening), as well as the final number of fertile tillers. This approach allowed the identification of four mutants with different tillering behaviour: in M07.5478 and M08.0589 tillering was increased at all stages, while M09.7479 had increased tiller numbers at heading and ripening, but not at tillering stage, and M16.3026 showed increased tiller number only at heading stage. These results suggest that genetic control of tillering at later developmental stages may at least in part be distinct from earlier stages. This idea is consistent with results from a recent genome-wide association study of tillering in a collection of 768 barley lines: tiller number at later time points was associated with more quantitative trait loci (QTLs) compared to early time points (Haaning et al. 2020). Mutants M07.5478 and M08.0589 were especially interesting as they started tillering early resulting in higher numbers of fertile tillers. Indeed, both mutants had increased values for biomass, SeedPl, SeedSp and SeedYPl. Interestingly, these lines also showed increased plant height compared to cv. Sebastian, in contrast to previously introduced mutants in which plant height was lower than wild-type (Franckowiak and Lundqvist 2002, 2013; Babb and Muehlbauer 2003; Druka et al. 2011; Ramsay et al. 2011; Okagaki et al. 2013; Mascher et al. 2014; Hussien et al. 2014; Marzec et al. 2016; Shaaf et al. 2019).

Analysis of correlation and PCA, indicated stronger relationships between tillering and biomass and yield related traits in the TA group compared to LA group. In general, TN mutants had also increased levels of yield. This is in contrast 
to high tillering mutant int- $m$ which exhibited reduced number of seeds compared to wild type (Liller et al. 2015).

With respect to LA mutants, our results show no major differences in yield components. While many studies have shown close relationships between leaf erectness and increased yield in cereals (Mantilla-Perez and Salas Fernandez 2017), this effect is largely dependent on improved radiation distribution at canopy level in high plant density conditions. In the current study agronomic evaluations were conducted on single plants grown in rows, which is not representative of the conditions in a plant stand. Further characterization of our LA mutants should be carried out at plot level under different planting densities.

In conclusion, promising mutant lines for both erect leaf angle and increased tiller number were reported in our study. Further investigations are needed to identify the underlying genes and gain insight into the molecular bases of these phenotypic effects.

Supplementary Information The online version contains supplementary material available at https://doi.org/10.1007/s42976-021-00178-6.

Authors' contributions SMA performed statistical analyses, drafted the paper. BAM revised the manuscript for important intellectual content. AJ provided genetic materials, revised the manuscript for important intellectual content. LR conceived and coordinated the project, complemented writing of the manuscript. SS designed the experiment, provided and analyzed the data, prepared the figures, and complemented writing of the manuscript. All authors read and approved the final version of the manuscript.

Funding Open access funding provided by Università degli Studi di Milano within the CRUI-CARE Agreement. We gratefully acknowledge funding from the FACCE ERA-NET BarPLUS project (ERANET FACCE SURPLUS grant 93 to LR and AJ). SS in part was supported by a post-doctoral fellowship by the University of Milan (assegno di ricerca di tipo A).

Availability of data and materials Plant materials described in the present work are available upon request. The author responsible for distribution is Agnieszka Janiak: agnieszka.janiak@us.edu.pl.

Code availability Non applicable.

\section{Declarations}

Conflict of interest The authors declare no conflict of interest.

Open Access This article is licensed under a Creative Commons Attribution 4.0 International License, which permits use, sharing, adaptation, distribution and reproduction in any medium or format, as long as you give appropriate credit to the original author(s) and the source, provide a link to the Creative Commons licence, and indicate if changes were made. The images or other third party material in this article are included in the article's Creative Commons licence, unless indicated otherwise in a credit line to the material. If material is not included in the article's Creative Commons licence and your intended use is not permitted by statutory regulation or exceeds the permitted use, you will need to obtain permission directly from the copyright holder. To view a copy of this licence, visit http://creativecommons.org/licenses/by/4.0/.

\section{References}

Ahloowalia BS, Maluszynski M, Nichterlein K (2004) Global impact of mutation-derived varieties. Euphytica 135:187-204

Aisawi KAB, Reynolds MP, Singh RP, Foulkes MJ (2015) The physiological basis of the genetic progress in yield potential of CIMMYT spring wheat cultivars from 1966 to 2009. Crop Sci 55:1749-1764

Babb S, Muehlbauer GJ (2003) Genetic and morphological characterization of the barley uniculm2 (cul2) mutant. Theor Appl Genet 106:846-857

Barnaby JY, Pinson SR, Chun J, Bui LT (2019) Covariation among root biomass, shoot biomass, and tiller number in three rice populations. Crop Sci 59:1516-1530

Bates D, Mächler M, Bolker B, Walker S (2015) Fitting linear mixedeffects models using lme4. J Stat Softw 67:1-48

Boe A (2007) Variation between two switchgrass cultivars for components of vegetative and seed biomass. Crop Sci 47:634-640

Braumann I, Dockter C, Beier S, Himmelbach A, Lok F, Lundqvist L, Skadhauge B, Stein N, Zakhrabekova S, Zhou R, Hansson M (2017) Mutations in the gene of the Ga subunit of the heterotrimeric $\mathrm{G}$ protein are the cause for the brachytic1 semidwarf phenotype in barley and applicable for practical breeding. Hereditas 155:10

Braumann I, Urban W, Preuáß A, Dockter C, Zakhrabekova S, Hansson M (2018) Semi-dwarf barley (Hordeum vulgare L.) brh2 and ari-l mutants are deficient in a U-box E3 ubiquitin ligase. Plant Growth Regul 86(2):223-234

Caldwell DG, McCallum N, Shaw P, Muehlbauer GJ, Marshall DF, Waugh R (2004) A structured mutant population for forward and reverse genetics in Barley (Hordeum vulgare L.). Plant $\mathrm{J}$ 40:143-150

Chono M, Honda I, Zeniya H, Yoneyama K, Saisho D, Takeda K, Takatsuto S, Hoshino T, Watanabe Y (2003) A semi-dwarf phenotype of barley $u z u$ results from a nucleotide substitution in the gene encoding a putative brassinosteroid receptor. Plant Physiol 133:1209-1219

Choudhury BJ (2000) A sensitivity analysis of the radiation use efficiency for gross photosynthesis and net carbon accumulation by wheat. Agric for Meteorol 101:217-234

Confalonieri R, Paleari L, Foi M, Movedi E, Vesely FM, Thoelke W, Agape C, Borlini G, Ferri I, Massara F, Motta R, Ravasi AR, Tartarini S, Zoppolato C, Baia LM, Brumana A, Colombo D, Curatolo A, Rossini L (2017) PocketPlant3D: analysing canopy structure using a smartphone. Biosyst Eng 164:1-12

Das MK, Fuentes RG, Taliaferro CM (2004) Genetic variability and trait relationships in switchgrass. Crop Sci 44:443-448. https:// doi.org/10.2135/cropsci2004.4430

Dawson IK, Russell J, Powell W, Steffenson B, Thomas WTB, Waugh R (2015) Barley: a translational model for adaptation to climate change. New Phytol 206:913-931. https://doi.org/ 10.1111/nph.13266

Dockter C, Gruszka D, Braumann I, Druka A, Druka I, Franckowiak J, Gough SP, Janeczko A, Kurowska M, Lundqvist J, Lundqvist U, Marzec M, Matyszczak I, Muller AH, Oklestkova J, Schulz B, Zakhrabekova S, Hansson M (2014) Induced variations in brassinosteroid genes define barley height and sturdiness, and expand the green revolution genetic toolkit. Plant Physiol 166:1912-1927 
Donald CT (1968) The breeding of crop ideotypes. Euphytica 17:385-403

Druka A, Franckowiak J, Lundqvist U, Bonar N, der Alexan- J et al (2011) Genetic dissection of barley morphology and development. Plant Physiol 155(2):617-627

FAO (2017) Food and Agriculture Organization (FAO) of the United Nations, Rome, Italy. Available at http://www.fao.org/3/i7454e/ i7454e.pdf. Accessed 26 May 2021

FAOSTAT data 2018. http://www.fao.org/faostat/en/\#data (consulted on May 26, 2021)

Foulkes MJ, Slafer GA, Davies WJ, Berry PM, Sylvester-Bradley R, Martre P et al (2011) Raising yield potential of wheat. III. Optimizing partitioning to grain while maintaining lodging resistance. J Exp Bot 62:469-486

Franckowiak JD, Lundqvist U (2002) New and revised barley genetic stock descriptions. Barley Genet Newslett 32:120

Franckowiak JD, Lundqvist U (2013) Descriptions of barley genetic stocks. Barley Genet Newsl 43:48-223

Gottwald S, Bauer P, Komatsuda T, Lundqvist U, Stein N (2009) TILLING in the two-rowed barley cultivar 'Barke' reveals preferred sites of functional diversity in the gene HvHoxl. BMC Res Notes 2:258

Haaning AM, Smith KP, Brown-Guedira GL, Chao S, Tyagi P, Muehlbauer GJ (2020) Natural genetic variation underlying tiller development in Barley (Hordeum vulgare L). G3 10:1197-1212

Hussien A, Tavakol E, Horner DS, Muñoz-Amatriaín M, Muehlbauer GJ, Rossini L (2014) Genetics of tillering in rice and barley. Plant Genome 7:1-20

Jia Q, Zhang X-Q, Westcott S, Broughton S, Cakir M, Yang J, Lance $\mathrm{R}, \mathrm{Li} \mathrm{C}$ (2011) Expression level of a gibberellin 20-oxidase gene is associated with multiple agronomic and quality traits in barley. Theor Appl Genet 122:1451-1460

Khush GS (2001) Green revolution: the way forward. Nat Rev Genet 2:815-822

Kuczynska A, Surma M, Adamski T, Mikoajczak K, Krystkowiak K, Ogrodowicz P (2013) Effects of the semi-dwarfing sdw1/denso gene in barley. J Appl Genet 54:381-390. https://doi.org/10.1007/ s13353-013-0165-x

Kumagai E, Hamaoka N, Araki T, Ueno O (2014) Dorsoventral asymmetry of photosynthesis and photoinhibition in flag leaves of two rice cultivars that differ in nitrogen response and leaf angle. Physiol Plant 151:533-543. https://doi.org/10.1111/ppl.12145

Lababidi S, Mejlhede N, Rasmussen SK, Backes G, Al-Said W, Baum M, Jahoor A (2009) Identification of barley mutants in the cultivar 'Lux' at the Dhn loci through TILLING. Plant Breed 128:332336. https://doi.org/10.1111/j.1439-0523.2009.01640.x

Li GH, Xue LH, Gu W, Yang CD, Wang SH, Ling QH, Qin X, Ding HF (2009) Comparison of yield components and plant type characteristics of high-yield rice between Taoyuan, a 'special eco-site' and Nanjing, China. Field Crops Res 112:214-221

Liller CB, Neuhaus R, Von Korff M, Koornneef M, Van Esse W (2015) Mutations in barley row type genes have pleiotropic effects on shoot branching. PLoS ONE 10:1-20

Lundqvist U (2014) Scandinavian mutation research in barley-a historical review. Hereditas 151:123-131

Mantilla-Perez MB, Salas Fernandez MG (2017) Differential manipulation of leaf angle throughout the canopy: current status and prospects. J Exp Bot 68(21-22):5699-5717

Marzec M, Gruszka D, Tylec P, Szarejko I (2016) Identification and functional analysis of the $\mathrm{HvDl} 14$ gene involved in strigolactone signaling in Hordeum vulgare. Physiol Plant 158:341-355

Mascher M, Jost M, Kuon JE, Himmelbach A, Aßfalg A, Beier S, Scholz U, Graner A, Stein N (2014) Mapping-by-sequencing accelerates forward genetics in barley. Genome Biol 15:R78

Mock JJ, Pearce RB (1975) An ideotype of maize. Euphytica 24(3):613-623
Munoz-Amatriain M, Cuesta-Marcos A, Hayes PM, Muehlbauer JG (2014) Barley genetic variation: implications for crop improvement. Brief Funct Genomics 13:341-350

Nachimuthu VV, Muthurajan R, Duraialaguraja S, Sivakami R, Pandian BA, Ponniah G, Sabariappan R (2015) Analysis of population structure and genetic diversity in rice germplasm using SSR markers: an initiative towards association mapping of agronomic traits in Oryza sativa. Rice 8(1):30

Okagaki RJ, Cho S, Kruger WM, Xu WW, Heinen S, Muehlbauer GJ (2013) The barley UNICULM2 gene resides in a centromeric region and may be associated with signaling and stress responses. Funct Integr Genomics 13:33-41

Ort DR, Merchant SS, Alric J, Barkan A et al (2015) Redesigning photosynthesis to sustainably meet global food and bioenergy demand. Proc Natl Acad Sci USA 112(28):8529-8536

Pankin A, Von-Korff M (2017) Co-evolution of methods and thoughts in cereal domestication studies: a tale of barley (Hordeum vulgare). Curr Opin Plant Biol 36:15-21

Parry MAJ, Reynolds M, Salvucci ME, Raines C, Andralojc PJ, Zhu XG, Price GD, Condon AG, Furbank RT (2011) Raising yield potential of wheat. II. Increasing photosynthetic capacity and efficiency. J Exp Bot 62:453-467. https://doi.org/10.1093/jxb/erq304

Peng S, Khush GS, Virk P, Tang Q, Zou Y (2008) Progress in ideotype breeding to increase rice yield potential. Field Crops Res 108(1):32-38

Ramsay L, Comadran J, Druka A, Marshall DF, Thomas WTB, MacAulay M, MacKenzie K, Simpson C, Fuller J, Bonar N, Hayes PM, Lundqvist U, Franckowiak JD, Close TJ, Muehlbauer GJ, Waugh R (2011) INTERMEDIUM-C, a modifier of lateral spikelet fertility in barley, is an ortholog of the maize domestication gene TEOSINTE BRANCHED 1. Nat Genet 43:169-172

Riaz A, Gul A (2015) Plant mutagenesis and crop improvement. In: Hakeem KR (ed) Crop production and global environmental issues. Springer, Cham, pp 181-210

RStudio Team (2020) RStudio: integrated development for R. RStudio, PBC, Boston. http://www.rstudio.com

Sakamoto T, Matsuoka M (2004) Generating high-yielding varieties by genetic manipulation of plant architecture. Curr Opin Biotechnol $15: 144-147$

Sakamoto T, Morinaka Y, Ohnishi T, Sunohara H, Fujioka S, UeguchiTanaka M, Mizutani M, Sakata K, Takatsuto S, Yoshida S, Tanaka H, Kitano H, Matsuoka M (2006) Erect leaves caused by brassinosteroid deficiency increase biomass production and grain yield in rice. Nat Biotechnol 24:105-109

Schreiber M, Barakate A, Uzrek N et al (2019) A highly mutagenised barley (cv. Golden Promise) TILLING population coupled with strategies for screening-by-sequencing. Plant Methods 15:99. https://doi.org/10.1186/s13007-019-0486-9

Seavers GP, Wright KJ (1999) Crop canopy development and structure influence weed suppression. Weed Res 39:319-328

Shaaf S, Bretani G, Biswas A, Fontana IM, Rossini L (2019) Genetics of barley tiller and leaf development. J Integr Plant Biol 61:226-256

Shearman VJ, Scott RK, Foulkes MJ (2005) Physiological processes associated with wheat yield progress in the UK. Crop Sci 185:175-185

Sreenivasulu N, Schnurbusch T (2012) A genetic playground for enhancing grain number in cereals. Trends Plant Sci 17:91-101. https://doi.org/10.1016/j.tplants.2011.11.003

Szarejko I, Maluszynski M (1999) High frequency of mutations after mutagenic treatment of barley seeds with $\mathrm{NaN} 3$ and $\mathrm{MNH}$ with application of inter-incubation germination period. Mutat Breed Newslett 44:28-30

Szarejko I, Szurman-Zubrzycka M, Nawrot M et al (2017) Creation of a TILLING population in barley after chemical mutagensesis with sodium azide and MNU. In: Jankowicz-Cieslak J, Tai 
TH, Kumlehn J, Till BJ (eds) Biotechnologies for plant mutation breeding. Springer, Berlin, pp 91-111

Szurman-Zubrzycka ME, Zbieszczyk J, Marzec M, Jelonek J, Chmielewska B, Kurowska MM et al (2018) HorTILLUS — a rich and renewable source of induced mutations for forward/reverse genetics and pre-breeding programs in barley (Hordeum vulgare L.). Front Plant Sci 9:216

Talamè V, Bovina R, Sanguineti MC, Tuberosa R, Lundqvist U, Salvi S (2008) TILLMore, a resource for the discovery of chemically induced mutants in barley. Plant Biotechnol J 6:477-485
Tavakol E, Okagaki R, Verderio G, Shariati JV, Hussien A (2015) The barley Uniculme4 gene encodes a BLADE-ON-PETIOLE-Like protein that controls tillering and leaf patterning. Plant Physiol 168(1):164-174

Wendt T, Holme I, Dockter C, Preuß A, Thomas W, Druka A, Waugh R, Hansson M, Braumann I (2016) HvDepl is a positive regulator of culm elongation and grain size in barley and impacts yield in an environment-dependent manner. PLoS ONE 11:e0168924 